PROCEEDINGS OF THE

AMERICAN MATHEMATICAL SOCIETY

Volume 132, Number 11, Pages 3349-3355

S 0002-9939(04)07439-8

Article electronically published on April 21, 2004

\title{
METRICS OF POSITIVE CURVATURE WITH CONIC SINGULARITIES ON THE SPHERE
}

\author{
A. EREMENKO
}

(Communicated by Juha M. Heinonen)

\begin{abstract}
A simple proof is given of the necessary and sufficient condition on a triple of positive numbers $\theta_{1}, \theta_{2}, \theta_{3}$ for the existence of a conformal metric of constant positive curvature on the sphere, with three conic singularities of total angles $2 \pi \theta_{1}, 2 \pi \theta_{2}, 2 \pi \theta_{3}$. The same condition is necessary and sufficient for the triple $\pi \theta_{1}, \pi \theta_{2}, \pi \theta_{3}$ to be interior angles of a spherical triangular membrane.
\end{abstract}

The following problem is classical [14, 15]. Let $S$ be a compact Riemann surface, $p_{1}, \ldots, p_{n}$ points in $S$, and $\theta_{1} \ldots, \theta_{n}$ positive numbers. Does there exist a conformal Riemannian metric of constant curvature $K$ with conic singularities at $p_{j}$ such that the total angle at $p_{j}$ is $2 \pi \theta_{j}$ ? A complete answer to this question is known when $K \leq 0$; see [7, 12 17, 18]. If $K \leq 0$, a unique metric exists if and only if this is not prohibited by the Gauss-Bonnet theorem. In Troyanov's papers [17, 18] the case of non-constant $K$ is also considered.

On the other hand, little is known for the case $K>0$. Troyanov [19] considered the case of two points on the sphere and showed that the necessary and sufficient condition in this case is $\theta_{1}=\theta_{2}$. It follows from the results of Troyanov in [17] that for any compact surface $S$, there exists a metric of constant positive curvature if the condition

$$
0<\chi(S)+\sum\left(\theta_{j}-1\right)<\min \left\{2,2 \min \theta_{j}\right\}
$$

is satisfied. Here the left inequality comes from the Gauss-Bonnet theorem while the right one is a technical restriction needed for Troyanov's method to work. If one assumes that all $\theta_{j} \in(0,1)$, and $S$ is the sphere, then condition (11) is actually necessary and sufficient [11].

In view of this situation, it seems useful to analyse the simple case of a metric of positive constant curvature on the sphere with three singularities. Here we give a complete solution for this case. To do this we return to the methods which were used before the PDE $\Delta u=K e^{u}$ was invoked; our arguments are based on the papers of Riemann [16].1. For modern expositions of this work, see, for example, [8, 21].

Received by the editors May 16, 2003 and, in revised form, July 22, 2003.

2000 Mathematics Subject Classification. Primary 53C45, 33C05.

The author was supported by NSF grant DMS 0100512 and by the Humboldt Foundation.

${ }^{1}$ Apparently the research of Klein, Schwarz and Poincaré which led to the discovery of the uniformization theorem was originally motivated by the study of ordinary linear differential equations, in particular, by the work of Riemann [16. Somewhat later, the Göttingen Royal Society suggested a different approach to the uniformization, a direct study of the non-linear PDE $\Delta u=e^{u}$,

(C)2004 American Mathematical Society 
The results show that for $K>0$ there are complicated restrictions in addition to the Gauss-Bonnet theorem.

In the generic case, when none of the prescribed angles is a multiple of $2 \pi$, our result (Theorem 1 below) was earlier obtained by Umehara and Yamada [20]. The method of these authors is somewhat indirect (they use a connection with surfaces of constant mean curvature 1 in the hyperbolic space) and they do not obtain an explicit condition in the case of integer $\theta_{j}$. In a recent preprint [6], results which are equivalent to our Theorems 1 and 2 are obtained by a pure geometric method, which is different from the analytic method used here. It seems that the proofs presented below are simpler. Conformal metrics of constant curvature on the sphere with three conic singularities were also studied by physicists [4, but they did not address the question of existence or uniqueness of such metrics.

We identify $S \backslash\left\{p_{3}\right\}$ with the complex plane $\mathbf{C}$ and assume without loss of generality that $K=1, p_{1}=0, p_{2}=1$ and $p_{3}=\infty$. Suppose that a conformal Riemannian metric of constant curvature 1 is given on $D=\mathbf{C} \backslash\{0,1\}$. Then every point $z \in D$ has a neighborhood which is isometric to an open set on the unit sphere $\overline{\mathbf{C}}[10$. The isometry is a conformal map, so we obtain a multi-valued locally univalent meromorphic function $f$ in $D$, whose monodromy belongs to the group of orientation-preserving isometries of $\overline{\mathbf{C}}$. This $f$ is sometimes called a "developing map" in geometric literature.

The converse is also true: given such a multi-valued function $f$, we define a conformal metric of constant curvature 1 in $D$ by the length element

$$
\lambda(z)|d z|=\frac{2\left|f^{\prime}(z)\right||d z|}{1+|f(z)|^{2}} .
$$

The behavior of $f$ at $\{0,1, \infty\}$ reflects the assumption that these points are conical singularities with total angles $2 \pi \theta_{1}, 2 \pi \theta_{2}, 2 \pi \theta_{3}$ : in a local coordinate $z$ near $p_{j}$ some fractional-linear transformation $g_{j}=L_{j} \circ f$ of $f$ has the form

$$
g_{j}(z)=z^{\theta_{j}} .
$$

It follows that $f$ satisfies a Schwarz differential equation

$$
\frac{f^{\prime \prime \prime}}{f^{\prime}}-\frac{3}{2}\left(\frac{f^{\prime \prime}}{f^{\prime}}\right)^{2}=R(z)
$$

where

$$
R(z)=\frac{1-\theta_{1}^{2}}{2 z^{2}}+\frac{\theta_{1}^{2}+\theta_{2}^{2}-\theta_{3}^{2}-1}{2 z(1-z)}+\frac{1-\theta_{2}^{2}}{2(z-1)^{2}} .
$$

The equation (2) defines a metric with the desired properties if and only if its monodromy group is conjugate to a subgroup of conformal isometries of the sphere.

It remains to find out when this is the case. This problem was considered in another context by Arnold and Krylov in [1, but they state its solution only when $\theta_{j} \in(0,1)$.

We begin by remarking that (2) is an equation with real coefficients. It follows that our function $f$ maps the upper half plane onto a circular triangle (membrane) $T$ that is a simply connected surface spread over the sphere whose boundary consists of three arcs of circles (each of these circles may be traced more than a full turn). We will show that the monodromy group is conjugate to a group of conformal isometries

which was proposed as a topic of a competition. It seems that the papers [14 15] were inspired by this competition. 
if and only if this triangle $T$ can be mapped by a fractional-linear transformation onto to a spherical (geodesic) triangle. In the opposite direction, if a geodesic triangular membrane $T$ is given, we can paste it together with its mirror image $T^{\prime}$ and obtain a surface homeomorphic to the sphere, with metric of positive curvature 1 and three conic singularities whose total angles are twice those of $T$.

It is well-known (since the work of Schwarz) that the monodromy group of the Schwarz equation (2) is the same as the projectivized monodromy group of the hypergeometric (Gauss) equation

$$
z(1-z) w^{\prime \prime}+(c-(a+b+1) z) w^{\prime}-a b w=0,
$$

where the parameters $a, b, c$ are related to the parameters $\theta_{j}$ in the following way:

$$
\begin{aligned}
& \pm \theta_{1}=1-c, \\
& \pm \theta_{2}=a-b, \\
& \pm \theta_{3}=c-a-b .
\end{aligned}
$$

1. Let us first consider the case that none of the numbers $\theta_{j}$ is an integer. Let $\Gamma^{\prime}$ be the group generated by reflections in the sides of the circular triangle $T$. Then the subgroup $\Gamma \subset \Gamma^{\prime}$ of index 2 consisting of orientation-preserving transformations coincides with the monodromy group of the equation (2). If $C_{1}$ and $C_{2}$ are two sides of $T$ with the common vertex $v$, then the group $\Gamma$ contains an elliptic transformation fixing $v$ and the other point $v^{\prime}$ where the circles containing $C_{1}$ and $C_{2}$ intersect. It is evident that the group $\Gamma$ is conjugate to a group of isometries of the sphere if and only if there exists a fractional-linear transformation which sends all three pairs $\left(v, v^{\prime}\right)$ to pairs of diametrically opposite points. This is the case if and only if $T$ is equivalent by a fractional-linear transformation to a triangle whose sides are geodesic.

Monodromy of the equation (3) was explicitly computed by Riemann 16]; this result is reproduced in many places, for example, in [8, 9, 13, 21]. It follows from the explicit formulas that (under the condition that none of the $\theta_{j}$ is an integer) this monodromy remains unchanged if a triple $\left(\theta_{1}, \theta_{2}, \theta_{3}\right)$ is replaced by a triple $\left( \pm \theta_{1}+m, \pm \theta_{2}+n, \pm \theta_{3}+k\right)$, where $(m, n, k)$ are integers with the property $m+n+k \equiv 0(\bmod 2)$. Let us call such triples of positive numbers equivalent. Every non-integer triple is equivalent to one and only one triple with the property

$$
0<\theta_{1}^{\prime}+\theta_{2}^{\prime} \leq 1, \quad 0<\theta_{2}^{\prime}+\theta_{3}^{\prime} \leq 1, \quad 0<\theta_{1}^{\prime}+\theta_{3}^{\prime} \leq 1 .
$$

It is easy to show that for every triple of positive numbers satisfying (15) there is a unique (up to a fractional-linear transformation) circular triangle with angles $\left(\pi \theta_{1}^{\prime}, \pi \theta_{2}^{\prime}, \pi \theta_{3}^{\prime}\right)$, and this triangle consists of one sheet (is a Jordan region on the sphere). Such a triangle $T$ is equivalent to a geodesic triangle if and only if the sum of its angles is greater than $\pi$. Thus we obtain

Theorem 1. If none of $\theta_{1}, \theta_{2}, \theta_{3}$ is an integer, then a conformal metric of constant positive curvature on the sphere with conic singularities of total angles $2 \pi \theta_{1}, 2 \pi \theta_{2}$ and $2 \pi \theta_{3}$ exists if and only if the unique equivalent triple with the property (5) satisfies $\theta_{1}^{\prime}+\theta_{2}^{\prime}+\theta_{3}^{\prime}>1$. Such a metric of curvature 1 is unique.

The uniqueness statement holds because a triple $\left(\theta_{1}, \theta_{2}, \theta_{3}\right)$ uniquely defines the right hand side $R$ of the Schwarz equation (2). A solution of the Schwarz equation with isometric monodromy is then defined up to a rotation of the sphere. 
2. Now we consider the case when at least one of the three numbers $\theta_{1}, \theta_{2}, \theta_{3}$ is an integer. We assume that

$$
\theta_{j} \neq 1, \quad j=1,2,3,
$$

because the case $\theta_{j}=1$ is covered by the result of Troyanov [19] mentioned in the beginning.

First of all, we have a necessary condition for the monodromy group to be a group of isometries, that no logarithms are present in the formal solutions of (3) at its singular points. (Presence of a logarithm leads to a parabolic transformation in the monodromy group, see, for example, [9.)

Without loss of generality, we consider the singularity of the equation (31) at zero and suppose that $N=\theta_{1}=1-c$ is a positive integer. The exponents at zero are 0 and $N$. The logarithms will be absent if a power series

$$
w=\sum_{k=0}^{\infty} a_{k} z^{k}, \quad a_{0} \neq 0,
$$

satisfies (3). Substituting this series into the equation, we obtain

$$
[k(k-1)+c k] a_{k}=[(k-1)(k-2)+(a+b+1)(k-1)+a b] a_{k-1}, \quad k=1,2, \ldots
$$

The coefficient in the left hand side is zero when $k=1-c=N$. So a power series solution of the form (6) exists if and only if the coefficient in the right hand side is zero for some $k=n \in[1, N]$, that is,

$$
(n-1)(n-2)+(a+b+1)(n-1)+a b=0
$$

or

$$
(n-1)(a+b+n-1)+a b=0 .
$$

Expressing $a, b$ in terms of $\theta_{2}, \theta_{3}$ and $N=\theta_{1}$, from (4), we obtain, after simple transformations, that either the sum or the difference of $\theta_{1}$ and $\theta_{2}$ equals $2 n-N-1$. In other words, one of the following equations holds:

$$
\left|\theta_{1} \pm \theta_{2}\right| \in[0, N-1] \text { is an integer of the opposite parity from } N \text {. }
$$

This condition is necessary for the monodromy of (2) to consist of isometries. Let us prove that it is also sufficient. If the angle at a vertex $v$ of the triangle $T$ is an integer multiple of $\pi$, and no logarithms are present in solutions, then two sides of $T$ meeting at $v$ belong to the same circle, so all sides of $T$ belong to two intersecting circles. But every such pair of circles can be mapped by a fractionallinear transformation onto a pair of great circles (by sending their two points of intersection to diametrically opposite points). So the group generated by reflections in sides of $T$ is conjugate to a group of isometries. Thus we obtain

Theorem 2. If $\theta_{1}$ is an integer, then a necessary and sufficient condition for the existence of a conformal metric of curvature 1 on the sphere, with three conic singularities of angles $2 \pi \theta_{1}, 2 \pi \theta_{2}, 2 \pi \theta_{3}\left(\theta_{j} \neq 1,1 \leq j \leq 3\right)$, is that either $\theta_{2}+\theta_{3}$ or $\left|\theta_{2}-\theta_{3}\right|$ is an integer $m$ of opposite parity from $\theta_{1}$, and $m \leq \theta_{1}-1$. This metric with prescribed conical points is unique when it exists.

Further remarks. 1 . If two of the $\theta_{j}$ are integers, and the condition in Theorem 2 is satisfied, then the third one is an integer as well. Then the necessary and sufficient condition becomes 

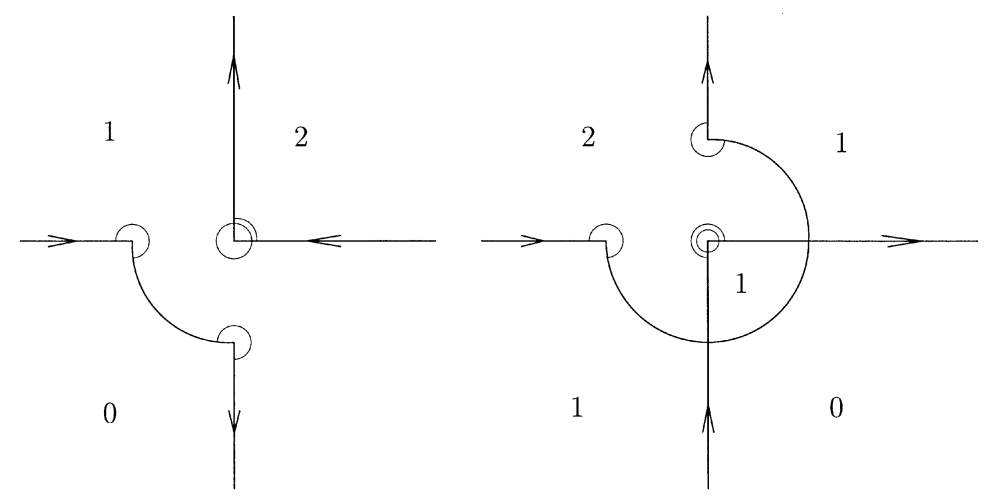

Figure 1. Spherical triangular membranes with angles $3 \pi / 2,3 \pi / 2,5 \pi / 2$ (left) and $3 \pi / 2,3 \pi / 2,7 \pi / 2$ (right) represented as surfaces spread over the sphere. The sides project into the great circles which are the coordinate axes and the unit circle. The covering numbers are shown for each region to help visualize the surface.

The sum of the three integers is odd, and each of them is less than the sum of the others.

The solution of the Schwarz equation in this case has trivial monodromy, and thus it is a rational function. It has critical points at 0,1 and $\infty$. The local degrees at these points are $\theta_{j}$, and the condition above is necessary and sufficient for the existence of such a rational function.

2. Our Theorems 1 and 2 also give necessary and sufficient conditions for the existence of a spherical triangular membrane with geodesic sides and prescribed angles. Let us give a precise definition.

A spherical triangular membrane is a bordered surface homeomorphic to the closed unit disc, with Riemannian metric of constant curvature 1, whose boundary consists of three geodesic arcs. It is clear how to define an interior angle of such a membrane. Spherical triangular membranes were considered by Klein in [9], but the following result does not seem to be explicitly stated there.

Theorem 3. There exists a spherical triangular membrane with interior angles $\pi \theta_{1}, \pi \theta_{2}, \pi \theta_{3}$ if and only if the three numbers $\theta_{1}, \theta_{2}$ and $\theta_{3}$ satisfy the conditions stated in Theorems 1 and 2.

It is somewhat hard to visualize spherical triangular membranes which have angles greater than $2 \pi$; the author thanks Mario Bonk for Figure 1.

3. In the case of four or more conic singularities, solution of the problem may not be unique. Indeed, consider the case when all total angles at the singularities are equal to $4 \pi$. Then solutions $f$ of the Schwarz equation are rational functions, and the problem is equivalent to finding rational functions with prescribed simple critical points. Let us call two rational functions equivalent if they are obtained from each other by post-composition with a fractional-linear transformation. It is 
known [5] that for every $2 d-2$ points in general position there exist

$$
u_{d}=\frac{1}{d}\left(\begin{array}{c}
2 d-2 \\
d-1
\end{array}\right), \quad \text { the } d \text {-th Catalan number, }
$$

equivalence classes of rational functions of degree $d$ with these critical points. Thus there are $u_{d}$ different conformal metrics of curvature 1 with conical singularities of total angle $4 \pi$ at $2 d-2$ given generic points. The smallest case of non-uniqueness occurs when $d=3$, so there are four conic singularities with total angles $4 \pi$.

Furthermore, for every odd $d \geq 3$, there are configurations of $2 d-2$ points symmetric with respect to the real line and such that the problem (with prescribed total angles of $4 \pi$ at each of these points) has no symmetric solutions with respect to the real line [3]. On the other hand, if all singularities belong to the real line, then all solutions of this problem are symmetric with respect to the real line [2].

\section{REFERENCES}

[1] V. I. Arnol'd and A. L. Krylov, Uniform distribution of points on the sphere and some ergodic properties of solutions of linear ordinary differential equations in the complex domain, Dokl. Akad. Nauk SSSR, 148 (1963) 9-12; English transl., Soviet Math. Dokl., 4 (1963) 1-5. MR 27:375

[2] A. Eremenko and A. Gabrielov, Rational functions with real critical points and the B. and M. Shapiro conjecture in real enumerative geometry, Ann. of Math., 155 (2002) 105-129. MR 2003c:58028

[3] A. Eremenko and A. Gabrielov, Counterexamples to pole placement by static output feedback, Linear Algebra and Appl., 351/352 (2002) 211-218. MR 2003f:93045

[4] A. Bilal and J-L. Gervais, Construction of constant curvature punctured Riemann surfaces with particle-scattering interpretation, J. Geom. Phys., 5 (1988) 277-304. MR 91e:81088

[5] L. Goldberg, Catalan numbers and branched coverings of the sphere, Adv. Math., 85 (1991) 129-144. MR 92b:14014

[6] M. Furuta and Y. Hattori, 2-dimensional singular spherical space forms, manuscript.

[7] M. Heins, On a class of conformal metrics, Nagoya Math. J., 21 (1962) 1-60. MR 26:1451

[8] E. Ince, Ordinary differential equations, Dover, NY, 1956. MR 6,65f

[9] F. Klein, Vorlesungen über die hypergeometrische Funktionen, Springer, Berlin, 1933. MR 84b:01060

[10] J. Liouville, Sur l'équation aux dérivées partielles $\partial^{2} \log \lambda / \partial u \partial v \pm 2 \lambda a^{2}=0$, J. de Math., 18 (1853) 71-72.

[11] F. Luo and G. Tian, Liouville equation and spherical convex polytopes, Proc. AMS, 116 (1992) 1119-1129. MR 93b:53034

[12] R. McOwen, Point singularities and conformal metrics on Riemann surfaces, Proc. AMS, 103 (1988) 222-224. MR 89m:30089

[13] É. Picard, Traité d'Analyse, t. III, Gauthier-Villars, Paris, 1896. MR 93i:01032c (reprint of 1928 ed.)

[14] É. Picard, De l'intégration de l'équation $\Delta u=e^{u}$ sur une surface de Riemann fermée, J. reine angew. Math., 130 (1905) 243-258.

[15] H. Poincaré, Fonctions fuchsiennes et l'équation $\Delta u=e^{u}$, J. de math. pures et appl., 5 (4) (1898) 137-230.

[16] B. Riemann, Beitrage zur Theorie der durch Gauss'sche Reihe $F(\alpha, \beta, \gamma, x)$ darstellbaren Funktionen, Ges. Math. Werke, 67-83; Vorlesungen über die hypergeometrische Reihe, Nachträge, III, 69-94. US edition: Dover, NY, 1953.

[17] M. Troyanov, Prescribing curvature on compact surfaces with conical singularities, Trans. AMS, 324 (1991) 793-821. MR 91h:53059

[18] M. Troyanov, Surfaces euclidiennes à singularités coniques, Enseign. Math., 32 (1986) 79-94. MR 87i:30079

[19] M. Troyanov, Metrics of constant curvature on a sphere with two conical singularities, Lect. Notes Math., 1410, Springer, NY, 1989, 296-308. MR 90m:53057 
[20] M. Umehara and K. Yamada, Metrics of constant curvature 1 with three conical singularities on 2-sphere, Illinois J. Math., 44 (2000) 72-94. MR 2001f:53072

[21] V. Varadarajan, Meromorphic differential equations. Expos. Math. 9 (1991) 97-188. MR 92i:32024

Department of Mathematics, Purdue University, West Lafayette, Indiana 47907

E-mail address: eremenko@math.purdue.edu 